\title{
Black-box warning for antidepressants
}

\section{Editorial}

On an average, U.S. FDA has approved one new antidepressant drug per year for the last 20 years. In spite of some initial breakthrough in the treatment of depression, it remained as the most common psychiatric illness. A result from the 2014 National survey on Drug Use and Health showed that rate of depression is especially higher in younger population. In $2014,11.4 \%$ of youths aged $12-17$ (2.8 million adolescents) had a major depressive episode in the past year. The 2014 percentage was higher than the percentages in 2004-2012. ${ }^{1}$

All currently approved antidepressants have very similar mechanism of action and most of them are 'me too' drugs. These antidepressants are believed to work by reversing the monoamine neurotransmitter deficiency in depressed patients. These medications usually increase the levels of serotonin, nor epinephrine, or dopamine in the brain by blocking the reuptake mechanism or enzyme inhibition. Adverse effects including suicidal ideation in younger population are major limitations of current antidepressants.

Although younger population is more affected by depression, all marketed antidepressants contain black-box warning for them. Black-box is the most serious type of warning given by the Food and Drug Administration (FDA) for prescription medication. The warning issued in 2004, states that antidepressants are associated with suicidal thinking, feeling, and behavior in young people. The warning was based on a series of meta-analyses of 295 individual clinical trials of antidepressants such as tricyclic antidepressants, monoamine oxidase inhibitors, and selective serotonin reuptake inhibitors. In 2007, FDA extended the warning for young adults aged 18-24. The warning also notes that children and adolescents taking selective serotonin reuptake inhibitor antidepressants should be closely monitored for adverse behavioral change.

The black-box warning for antidepressant gave rise to controversies among clinical practitioners. In the FDA review, the rate of suicidal thinking in children receiving antidepressants was $4 \%$, in comparison to $2 \%$ suicidal thinking in children taking placebo. None of the children actually committed suicide. A comprehensive review of pediatric clinical trials conducted between 1988 and 2006 suggested that the benefits of antidepressants may outweigh their risks to children and adolescents. ${ }^{2}$ More recently, a retrospective study revealed the likely impact of this FDA warning on US population. It was found that black-box warning was followed by a significant drop of antidepressant use and increased suicide attempts in general population. ${ }^{3}$ Most likely the physicians became more restrictive to prescribe the antidepressants or the patients became reluctant to disclose depression symptoms in the wake of FDA advisory.

Moreover, the black-box warning was issued for antidepressants that had very similar mechanism of action: regulation of monoamine

\author{
Volume 2 Issue I - 2016
}

\author{
Monzurul Amin Roni \\ Department of Pharmaceutical Sciences, Hampton University \\ School of Pharmacy, USA
}
Correspondence: Monzurul Amin Roni, Department of Pharmaceutical Sciences, Hampton University School of Pharmacy, Kittrell Hall, Hampton,VA 23693, USA, Tel + I 757-728-6404, Fax + I 757-727-5840, Email monzurul.roni@hamptonu.edu

Received: August 19, 2016 | Published: August 22, 2016

neurotransmitters such as serotonin, nor epinephrine, and dopamine. Recent advances in this field indicate that other targets such as regulation of glutamate neurotransmission may lead to discovery and development of better and possibly safer antidepressants. These antidepressants with mechanism other than monoamine neurotransmission are currently in advanced phases of clinical trials. It is not clear whether antidepressants with novel mechanisms should also receive black-box warning label. Considering the controversy surrounding this black-box warning over the past decade, FDA advisory panel should reevaluate the justification of black-box warning for all antidepressants.

\section{Acknowledgements}

None.

\section{Conflict of interest}

The author declares no conflict of interest.

\section{References}

1. Sarra L Hedden, Joel Kennet, Rachel Lipari, et al. Behavioral health trends in the United States: Results from the 2014 National Survey on Drug Use and Health. Center for Behavioral Health Statistics and Quality. 2015.

2. Bridge JA, Iyengar S, Salary CB, et al. Clinical response and risk for reported suicidal ideation and suicide attempts in pediatric antidepressant treatment: a meta-analysis of randomized controlled trials. JAMA. 2007;297(15):1683-1696.

3. Lu CY, Zhang F, Lakoma MD, et al. Changes in antidepressant use by young people and suicidal behavior after FDA warnings and media coverage: quasi-experimental study. BMJ. 2014;348:g3596. 